\title{
INSTANTANEOUS AND INTEGRAL POWER EQUATIONS OF NONSINUSOIDAL 3-PHASE PROCESSES
}

\begin{abstract}
Purpose. To identify the mathematical relationship between the instantaneous powers (classical and vectorial) and integral powers in non-sinusoidal mode and to get complex form of instantaneous powers in 3-phase 4-wire power supply in terms of the spectral approach. Methodology. We have applied the vector approach with one voice allows you to analyze the energy characteristics of 3-phase power supply circuits (for 4-wire and 3-wire circuits) in sinusoidal and non-sinusoidal mode, both the time domain and frequency domain. We have used 3-dimensional representation of the energy waveforms with the complex multi-dimensional Fourier series. Results. For 4-wire network with a non-sinusoidal (regardless of their symmetry) processes, we have developed the mathematical model one-dimensional representations of the complex form for the active (scalar) instantaneous power (IP) and 3 dimensional form (inactive) vectorial IP. It is possible to obtain two dual integral power equations for complex scalar and vector integrated power of non-sinusoidal modes. The power equations generalize generalizes the equations of sinusoidal modes for 4wire network. Originality. In addition to the classification of energy local regimes in the time domain for the first time we spent the classification of non-sinusoidal modes in the spectral region and showed the value and importance of the classification of regimes based on the instantaneous powers. Practical value. The practical value the obtained equations is the possibility of their use for improving the quality of electricity supply and the quality electricity consumption. References 3, figures 3.

Key words: three-phase circuit, classical instantaneous power, vector instantaneous power, complex 3-dimensional Fourier series, active and reactive power, complex vector power, apparent power, complex pulsation power, power equation, unbalanced mode, non-sinusoidal mode, 3-phasor.
\end{abstract}

Для 3- фазной схемы электроснабжения рассмотрены несинусоидльные режсмы, классифицируемые скалярной и векторной мгновенными мощностями (ММ). В рамках временного и спектрального подходов теории мощности получены комплексные формы активной (скалярной) ММ и (неактивной) векторной ММ. Для 4- проводной сети получены уравнения мощности комплексных скалярных и комплексных векторных мощностей несинусоидальных режимов. Уравнения мощностей обобщают соответствующие уравнения синусоидальных несимметричных режимов в 4- проводной сети. Библ. 3, рис. 3.

Ключевые слова: трехфазная цепь, классическая мгновенная мощность, векторная мгновенная мощность, комплексный 3-мерный ряд Фурье, активная и реактивная мощность, комплексная векторная мощность, кажущаяся мощность, комплексная мощность, уравнение мощности, несбалансированный режим, несинусоидальный режим, трехмерный комплексный коэффициент.

Introduction. Non-ideal (active-reactive, nonsymmetrical and nonlinear) load consumes not only electrical energy (EE) of active power but and EE of non-active components of apparent power (AP). For a number of such loads, consumption of $\mathrm{EE}$ of non-active components is caused by technological reasons and guarantees a longterm normal mode of the non-ideal (distorting) load operation. Non-active components of the AP cause additional losses in the power network making worse the power supply quality but they is not accounted and rested of.

An effective solution of the problem of the losses decrease and the EE account precision's increase is joint utilization of compensating devices (CD) and EE accounting tools. Existing accounting tools measure EE caused by the symmetry and linearity of the load's elements. Non-active TP components caused by the non-symmetry and nonlinearity of active-reactive load's element are not measured and accounted. Compensation, measurement and account of AP components are coupled, supplementing each other problems which from different economical points of view solve a problem of the effective EE consumption and should be solved in the frame of the general power theory $(\mathrm{PT})$ at real conditions of failure of the symmetry and the sinusoidality of the supply and consumption mode.

Problem definition. Increasing theoretical and practical interest for the PT definitions, interpretations of the reactive power conception interpretations, physical sense search, ambiguity of the apparent power determination in multiphase systems, the problem difficulty have been led to the creation of various PT «schools» (partial bibliography is presented in [1]). For non-sinusoidal multiphase processes two alternative approaches of investigation and analysis of the PT concepts are used: spectral (Budeanu, Quade, Pukhov, Emanuel, Czarnecki, Shidlovsky, Kuznetsov, Lev-Ari and Stanković, etc.) and temporar (Buchholz, Fryze, Depenbrock, Demirchan, Maevsky, Nabae and Akagi, Willems, Watanabe and Aredes, Tolber, Tonkal and Novoseltsev, etc.).

The temporal method of the analysis is based on a special expansion of the 3-phase current on orthogonal components. One of components of such a special expansion determines the active current which after the compensation remains in the source's network and guarantees supply of EE of active power. Temporal method used two approaches to the 3 -phase processes investigation. The first approach considers 3-phase processes as 3D curves on the averaging interval, is connected with generalization on the multiphase processes of the Fryze method and uses integral powers (IntP). The second one is based on instantaneous energetic characteristics: classical (scalar) instantaneous power (IP) and new vectorial IP (a cross-vector theory). This approach has a practical value and resulted in the development of so-called active filters. However, even for the sinusoidal mode mathematical connections between new IP and classical integral powers of the spectral approach did not finally determine [1].

The aim of the work is determination of the connection between the IP and integral powers and obtaining the (C) Iu.A. Sirotin, T.S. Ierusalimova 
complex form of the scalar and vectorial IP for nonsinusoidal modes classification in the 3-phase 4-wire electric power supply circuit in the terms of the spectral approach.

The used methodology is based on the vector approach with from the common point of view permits to analyze energetic characteristics both for 4-wire and for 3-wire circuits, both at sinusoidal and at non-sinusoidal mode, and both in time and in frequency domain.

Scalar (classical) IP. At the consideration of a 3phase 4-wire network we assume that voltage in phase are measured relatively the neutral terminal (Fig. 1). In every time moment $t$ voltage instantaneous values (IV) (regarding «neutral terminal's» wire) and current IV in phases are considered as 3D vectors of an arithmetic 3D space $R^{(3)}$

$$
\boldsymbol{u}(t)=\left[\begin{array}{lll}
u_{a}(t) & u_{b}(t) & u_{c}(t)
\end{array}\right]^{\tau}, \boldsymbol{i}(t)=\left[\begin{array}{lll}
i_{a}(t) & i_{b}(t) & i_{c}(t)
\end{array}\right]^{\tau},
$$

here in after $\tau$ is the sign of transposition.

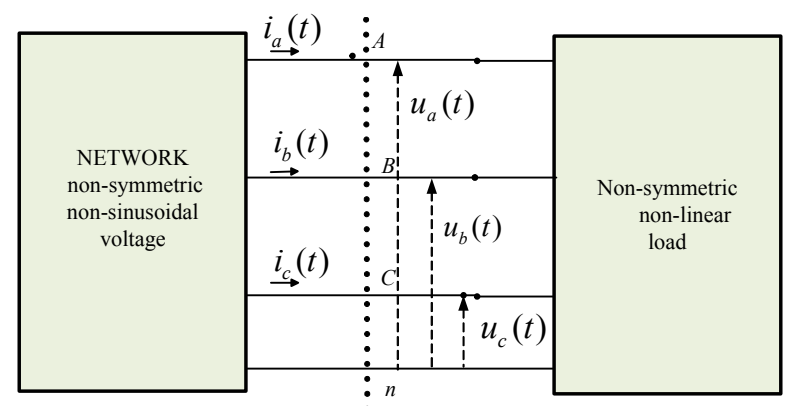

Fig. 1. Energetic processes in 4-wire electric power supply circuit

Determination of the norm in the 3D arithmetic space $R^{(3)}$ in each moment of time determines the norm of the vector of the current and voltage IV

$$
\begin{gathered}
|\boldsymbol{u}|=|\boldsymbol{u}(t)|=\sqrt{\boldsymbol{u}^{\tau} \boldsymbol{u}}=\sqrt{u_{a}(t)^{2}+u_{b}(t)^{2}+u_{b}(t)^{2}}, \\
|\boldsymbol{i}|=|\boldsymbol{i}(t)|=\sqrt{\boldsymbol{i}^{\tau} \boldsymbol{i}}=\sqrt{i_{a}(t)^{2}+i_{b}(t)^{2}+i_{b}(t)^{2}} .
\end{gathered}
$$

A local state of the energetic mode in the $3 \mathrm{D}$ section $<A, B, C>$ is characterized by the (classical) IP

$$
p(t)=u_{a}(t) i_{a}(t)+u_{b}(t) i_{b}(t)+u_{c}(t) i_{c}(t)=\frac{d W}{d t} .
$$

IP is determined as a sum of pair-wise products of current and voltage IV of three phases and determines the velocity of the energy transfer $W=W(t)$ in the section $<A, B, C>$. As appears from (4), at each moment of time the IP equals to the scalar product (SP) of vectors (1) in the space $R^{(3)}$

$$
p(t)=(\boldsymbol{i}, \boldsymbol{u})=\boldsymbol{i}^{\tau} \boldsymbol{u}=\left[\begin{array}{lll}
i_{a}(t) & i_{b}(t) & i_{c}(t)
\end{array}\right] \cdot\left[\begin{array}{l}
u_{a}(t) \\
u_{b}(t) \\
u_{c}(t)
\end{array}\right] .
$$

Vectorial IP and an equation of IP. Product of norms of the vectors (2), (3) determines the apparent (total) IP of the energetic mode

$$
s(t)=|\boldsymbol{i}(t)| \cdot|\boldsymbol{u}(t)|=i(t) \cdot u(t) .
$$

In the $3 \mathrm{D}$ space $R^{(3)}$ for any couple vectors the Cauchy-Schwarz inequality is true that for the vectors (1) inequality gives an implication

$$
\left|\boldsymbol{i}^{\tau}(t) \boldsymbol{u}(t)\right| \leq|\boldsymbol{i}(t)| \cdot|\boldsymbol{u}(t)| \Rightarrow p(t) \leq s(t) .
$$

The vector IP is a vector of the space $R^{(3)}$ which is introduces as the vector product (VP) of IV of vectors (1) of currents and voltages [1]

$$
\boldsymbol{q}(t)=\boldsymbol{i} \times \boldsymbol{u}=[\underbrace{i_{b} u_{c}-i_{c} u_{b}}_{q_{a}} \underbrace{i_{c} u_{a}-i_{a} u_{c}}_{q_{b}} \underbrace{i_{a} u_{b}-i_{b} u_{a}}_{q_{c}}]^{\tau}
$$

The Gram determinant [2] (composed from pairwise scalar products of the current and voltage IV vectors) equals to square of the vectorial IP (8)

$$
\left[\begin{array}{ll}
\boldsymbol{i}^{\tau} \boldsymbol{i} & \boldsymbol{i}^{\tau} \boldsymbol{u} \\
\boldsymbol{i}^{\tau} \boldsymbol{u} & \boldsymbol{u}^{\tau} \boldsymbol{u}
\end{array}\right]=[\underbrace{\boldsymbol{i} \times \boldsymbol{u}}_{\boldsymbol{q}(t)}]^{\tau}[\underbrace{\boldsymbol{i} \times \boldsymbol{u}}_{\boldsymbol{q}(t)}]=|\boldsymbol{q}(t)|^{2} .
$$

The geometrical sense of the Gram determinant - the «square of the area of the parallelogram built by current and voltage vectors» is shown in Fig. 2.

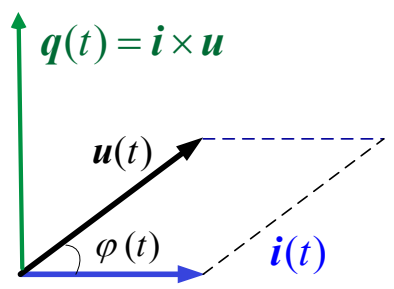

Fig. 2. Current vector, voltage vector, and the vector IP

Area of such an «instantaneous» parallelogram equals to

$$
q(t)=|\boldsymbol{i}(t)| \cdot|\boldsymbol{u}(t)| \cdot|\sin \varphi(t)|=s(t) \cdot|\sin \varphi(t)|,
$$

here $\varphi(t)$ is the instantaneous angle between vectors (1) in the space $R^{(3)}$ at the moment of time $t$.

The area of the parallelogram is equal to zero if vectors generating it are parallel (collinear, $\boldsymbol{i} \| \boldsymbol{u}$ ) when the apparent IP equals to the scalar (classical) IP. Therefore, norm of the VP of the current and voltage is interpreted as non-active IP. To underline this interpretation, the scalar (classical) IP (5) is named as active IP. Expansion (9) is invariant regarding the exchange of the vectors $\boldsymbol{i}$ and $\boldsymbol{u}$, but $\boldsymbol{i} \times \boldsymbol{u}=-\boldsymbol{u} \times \boldsymbol{i}$. In this paper (as in [1]) vectorial (nonactive) IP is determined in the correspondence with (8). Vectors $\boldsymbol{i}, \boldsymbol{u}, \boldsymbol{i} \times \boldsymbol{u}$ create a right-hand system.

The Gram determinant at each moment of time quadratically complements the scalar IP to the total (apparent) IP (6)

$$
\underbrace{\left(\boldsymbol{i}^{\tau} \boldsymbol{i}\right)}_{i^{2}(t)} \underbrace{\left(\boldsymbol{u}^{\tau} \boldsymbol{u}\right)}_{u^{2}(t)}=\underbrace{\left(\boldsymbol{i}^{\tau} \boldsymbol{u}\right)}_{p(t)})^{2}+[\underbrace{[\boldsymbol{i} \times \boldsymbol{u}}_{\boldsymbol{q}(t)}]^{\tau}[\boldsymbol{i} \times \boldsymbol{u}]
$$

and gives an equation for instantaneous powers

$$
s^{2}(t)=p^{2}(t)+q^{2}(t)
$$

which is illustrated by Fig. 3 .

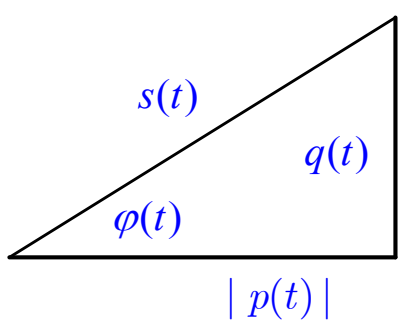

Fig. 3. A triangle of instantaneous powers

In the triangle of the IP two cathetus correspond to active and non-active instantaneous powers. If the nonactive IP is determined by $\sin \varphi(t)$, than the active IP is determined by $\cos \varphi(t)$ 


$$
p(t)=\left(\boldsymbol{i}^{\tau} \boldsymbol{u}\right)=\underbrace{|\boldsymbol{i}| \boldsymbol{u} \mid}_{s(t)} \cdot \mid \underbrace{\frac{\left(\boldsymbol{i}^{\tau} \boldsymbol{u}\right)}{|\boldsymbol{i} \| \boldsymbol{u}|}}_{\cos \varphi(t)}=s(t) \cdot \cos \varphi(t) .
$$

The angle $\varphi(t)$ in the triangle of the IP equals to the angle between current and voltage vectors introduced earlier. If the active IP (4) characterizes the effectiveness of the energetic mode, than the vector IP (13) characterizes the losses of the energetic mode.

Steady-state pulsed and unbalanced energetic mode. A steady-state energetic mode in a 3-phase section $<A, B, C>$ is determined by $3 \mathrm{D} T$-periodic curves of the current and voltage processes (waveforms):

$$
\boldsymbol{u}(t)=\boldsymbol{u}(t+T), \boldsymbol{i}(t)=\boldsymbol{i}(t+T) .
$$

For $T$-periodic processes the (integral) average IP is correctly determined, and the variable component is inambiguously extracted

$$
P=\bar{p}=\frac{1}{T} \int_{v}^{v+T} p(t) d t, \quad p(t)=\bar{p}+\widetilde{p}(t) .
$$

If IP has no variable (pulsed) component $\tilde{p}(t) \equiv 0$, the mode is non-pulsed. In the general case $\tilde{p}(t)=p(t)-\bar{p} \neq 0$ and the steady mode is pulsed.

Like (15) in the vector IP it is possible to extract vector components: constant and variable ones

$$
\overline{\boldsymbol{q}}=\frac{1}{T} \int_{v}^{v+T} \boldsymbol{q}(t) d t ; \quad \boldsymbol{q}(t)=\overline{\boldsymbol{q}}+\widetilde{\boldsymbol{q}}(t)
$$

A mode at which the vectorial IP has no variable component $\widetilde{\boldsymbol{q}}=\widetilde{\boldsymbol{q}}(t) \equiv 0$ is named as a balanced mode [1].

The mode is really balanced if the vectorial IP (nonactive IP) identically equal to zero

$$
\boldsymbol{q}(t) \equiv 0 \Leftrightarrow(\overline{\boldsymbol{q}}=0) \&(\widetilde{\boldsymbol{q}}(t) \equiv 0) .
$$

As a result, the mode is really balanced $(q(t) \equiv|\boldsymbol{q}(t)| \equiv 0)$ if at each moment (identically) current and voltage vectors (1) are parallel in the arithmetic 3D space $R^{(3)}$

$$
q(t) \equiv 0 \Leftrightarrow \boldsymbol{i} \| \boldsymbol{u} \Leftrightarrow \boldsymbol{i}(t)=y(t) \boldsymbol{u}(t) .
$$

The scalar quantity $y(t)$ (it has dimensionality of the conductivity) is not obligatory a constant.

So, a couple of instantaneous characteristics - scalar (5) and vectorial (8) ones - characterize the local energetic mode in the section $\langle A, B, C\rangle$.

Spectral analysis of the periodic processes of the finite energy. A set of $3 \mathrm{D}$ (3-phase) $T$-periodic vector curves

$$
\boldsymbol{x}(t)=\left[\begin{array}{lll}
x_{a}(t) & x_{b}(t) & x_{c}(t)
\end{array}\right]^{\tau},
$$

with finite-average quadratic value

$$
\|\boldsymbol{x}\|^{2}=\overline{\boldsymbol{x}}^{2}=\frac{1}{T} \int_{v}^{v+T} \boldsymbol{x}(t)^{\tau} \boldsymbol{x}(t) d t<\infty
$$

creates a Gilbert infinite-dimensional space of 3D curves of the «finite energy»

$$
L_{2}^{(3)}(T)=\{\boldsymbol{x}(t), \quad t \in(v, v+T):\|\boldsymbol{x}\|<\infty\} .
$$

For 3-phase vector curves $\boldsymbol{x}(t), \boldsymbol{y}(t) \in L_{2}^{(3)}(T)$ a scalar product is determined

$$
<\boldsymbol{x}, \boldsymbol{y}>=\frac{1}{T} \int_{v}^{v+T} \boldsymbol{x}(t)^{\tau} \boldsymbol{y}(t) d t=\frac{1}{T} \int_{v}^{v+T}(\boldsymbol{x}(t), \boldsymbol{y}(t)) d t
$$

as integral average scalar product of IV in 3D space $R^{(3)}$. The Cauchy-Schwarz inequality is true [2]

$$
<\boldsymbol{x}, \boldsymbol{y}\rangle \leq\|\boldsymbol{x}\| \cdot\|\boldsymbol{y}\| .
$$

The $T$-periodic curve $\boldsymbol{x}(t)=\boldsymbol{x}(t+T)$ is expanded into the functional Fourier series of $3 \mathrm{D}$ harmonic components (sinusoidal, cosinusoidal, complex, etc.)

$$
\boldsymbol{x}(t)=\boldsymbol{x}_{0}(t)+\boldsymbol{x}_{1}(t)+\boldsymbol{x}_{2}(t)+\ldots+\boldsymbol{x}_{k}(t)+\ldots
$$

For the complex Fourier series a $3 \mathrm{D}$ vector harmonic $\boldsymbol{x}_{k}(t) \in L_{2}^{(3)}(T)$ of the $k$-th order

$$
\boldsymbol{x}_{k}(t)=\sqrt{2} \mathfrak{R} e\left[\boldsymbol{X}_{k} e^{j k \omega t}\right],(T \omega=2 \pi),
$$

is calculated by using a $3 \mathrm{D}$ complex coefficient (3-phasor)

$$
\boldsymbol{X}_{k}=\frac{\sqrt{2}}{T} \int_{v}^{v+T} \boldsymbol{x}(t) e^{-j k \omega t},(k=0,1,2, \ldots),
$$

$\boldsymbol{X}_{k}=\left[\begin{array}{lll}\dot{X}_{a, k} & \dot{X}_{b, k} & \dot{X}_{c, k}\end{array}\right]^{\tau}$ is the 3D vector with complex coordinates, $\mathfrak{R} e(\dot{z})$ is the real part of the complex number $\dot{z}$.

A set of 3-phasors composes a $3 \mathrm{D}$ complex space $C^{(3)}$ with complex scalar product [1]

$$
(\boldsymbol{X}, \boldsymbol{Y})=\boldsymbol{X}^{\tau} \boldsymbol{Y}^{*}
$$

* is the symbol of complex conjugation (CC). Further let's suppose that the constant component in the complex Fourier series is absent $\left(\boldsymbol{X}_{0}=0\right)$

$$
\boldsymbol{x}(t)=\sum_{k=1}^{\infty} \boldsymbol{x}_{k}(t)=\sum_{k=1}^{\infty} \underbrace{\sqrt{2} \mathfrak{R} e\left[\boldsymbol{X}_{k} e^{j k \omega t}\right]}_{\boldsymbol{x}_{k}(t)} .
$$

The complex form of the scalar IP. The spectral analysis of the $T$-periodical energetic processes of the current and voltage is used their representation by the complex Fourier series

$$
\begin{aligned}
& \boldsymbol{u}(t)=\sqrt{2} \mathfrak{R} e \sum_{k \geq 1} \boldsymbol{U}_{k} e^{j k \omega t}, \\
& \boldsymbol{i}(t)=\sqrt{2} \mathfrak{R} e \sum_{k \geq 1} \boldsymbol{I}_{k} e^{j k \omega t} .
\end{aligned}
$$

The Euler formula [2] represents components of the 3-curves of voltage (29) and current (30) expansion by using the $\mathrm{CC}$ of the 3 -phasor

$$
\begin{aligned}
& \boldsymbol{i}_{k}(t)=\frac{1}{2}\left[\boldsymbol{I}_{k} e^{j k \omega t}+\boldsymbol{I}_{k}^{*} e^{-j k \omega t}\right], \\
& \boldsymbol{u}_{k}(t)=\frac{1}{2}\left[\boldsymbol{U}_{k} e^{j k \omega t}+\boldsymbol{U}_{k}^{*} e^{-j k \omega t}\right] .
\end{aligned}
$$

In view of the scalar product's linearity, the IP equals to the sum of partial scalar IP's of the vector harmonics of the current of the $k$-th order and of the voltage of the $m$-th order

$$
p(t)=(\boldsymbol{i}, \boldsymbol{u})=\boldsymbol{i}^{\tau} \boldsymbol{u}=\sum_{k, m} \underbrace{\boldsymbol{i}_{k}(t)^{\tau} \boldsymbol{u}_{m}(t)}_{p_{k, m}(t)}=\sum_{k, m} p_{k, m}(t) .
$$

Representations (31), (32) of the vector harmonics by using the 3-phasor and its $\mathrm{CC}$ for the IV of the product of the vector harmonics of the current of the $k$-th order and of the voltage of the $m$-th order give an identity

$$
p_{k, m}(t)=\mathfrak{R} e[\underbrace{\boldsymbol{I}_{k}^{\tau} \boldsymbol{U}_{m}^{*}}_{\dot{N}_{k-m}} e^{j(k-m) \omega t}+\underbrace{\boldsymbol{I}_{k}^{\tau} \boldsymbol{U}_{m}}_{\dot{N}_{k+m}} e^{j(k+m) \omega t}] .
$$


If the current and voltage harmonics have the same order $(m=k)$ their scalar IP has both constant and variable components

$$
p_{k}(t)=\mathfrak{R} e[\underbrace{\boldsymbol{U}_{k}^{\tau} \boldsymbol{I}_{k}^{*}}_{\dot{S}_{G_{k}}}+\underbrace{\boldsymbol{I}_{k}^{\tau} \boldsymbol{U}_{k}}_{\dot{N}_{k}} e^{j 2 k \omega t}] .
$$

The scalar IP (4) is represented by the current and voltage 3-phasors as

$$
\begin{aligned}
& p(t)=\mathfrak{R} e\left\{\sum_{k \geq 1}\left[\dot{S}_{G_{k}}+\dot{N}_{k} e^{j 2 k \omega t}\right]\right\}+ \\
& +\mathfrak{R} e\left\{\sum_{k \neq m}\left[\dot{N}_{k-m} e^{j(k-m) \omega t}+\dot{N}_{k+m} e^{j(k+m) \omega t}\right]\right\}
\end{aligned}
$$

and has constant and variable (pulsed) components.

The constant component

$$
\bar{p}=\sum_{k \geq 1} \Re e\left[\dot{S}_{G_{k}}\right]=\sum_{k \geq 1} P_{k}=P
$$

equals to active (average) power of the non-sinusoidal mode and is represented as the real part of the complex (geometrical) power of all harmonics

$$
\dot{S}_{G}=\sum_{k \geq 1} \underbrace{\boldsymbol{U}_{k}^{\tau} \boldsymbol{I}_{k}^{*}}_{\dot{S}_{G_{k}}}=\sum_{k \geq 1} \dot{S}_{G_{k}}=P+j Q .
$$

The complex (geometrical) power (38) equals to the sum of harmonic components' complex powers

$$
\dot{S}_{G_{k}}=\boldsymbol{U}_{k}^{\tau} \boldsymbol{I}_{k}^{*}=\left(\boldsymbol{U}_{k}, \boldsymbol{I}_{k}\right)=P_{k}+j Q_{k},=S G_{k} \cdot e^{j \varphi_{k}} .
$$

The complex power (39) of the vector harmonics of the current and voltage of the $k$-th order equals to the complex scalar product (27) of the 3-phasor of the voltage and the 3-phasor of the current of the $k$-th order in the complex 3D space $C^{(3)}$.

The imaginary part of the complex power (38)

$$
Q=\mathfrak{I} m \sum_{k \geq 1} \underbrace{\boldsymbol{U}_{k}^{\tau} \boldsymbol{I}_{k}^{*}}_{\dot{S}_{G_{k}}}=\sum_{k \geq 1} \underbrace{\mathfrak{I} m\left[\boldsymbol{U}_{k}^{\tau} \boldsymbol{I}_{k}^{*}\right]}_{Q_{k}}=\sum_{k \geq 1} Q_{k}
$$

determines the non-sinusoidal mode's reactive power of the 3-phase section $\langle A, B, C>$ and gives the generalization of the reactive power by Budeanu to 3-phase processes

$$
Q=\sum_{k \geq 1} \underbrace{\mathfrak{I} m\left[\boldsymbol{U}_{k}^{\tau} \boldsymbol{I}_{k}^{*}\right]}_{Q_{k}}=\sum_{k \geq 1} Q_{k}=\sum_{k \geq 1} S Q_{k} \sin \varphi_{k} .
$$

Complex pulsation powers of the scalar IP:

- of the harmonics of the $k$-th order of the even frequency $2 k \omega$

$$
\dot{N}_{k}=\boldsymbol{I}_{k}^{\tau} \boldsymbol{U}_{k} ;
$$

- of the harmonics of the $k$-th order and $m$-th order of the sum and difference frequency

$$
\dot{N}_{k+m}=\boldsymbol{I}_{k}^{\tau} \boldsymbol{U}_{m}, \dot{N}_{k-m}=\boldsymbol{U}_{k}^{\tau} \boldsymbol{I}_{m}^{*}
$$

determined the transient (pulsed) component of the scalar IP

$$
\begin{aligned}
& \tilde{p}(t)=\mathfrak{R} e\left\{\sum_{k \geq 1} \dot{N}_{k} e^{j 2 k \omega t}\right\}+ \\
& +\mathfrak{R} e\left\{\sum_{k \neq m}\left[\dot{N}_{k-m} e^{j(k-m) \omega t}+\dot{N}_{k+m} e^{j(k+m) \omega t}\right]\right\} .
\end{aligned}
$$

If three types of the complex powers equal to zero

$$
\dot{N}_{k}=\dot{N}_{k+m}=\dot{N}_{k-m}=0,(k, m=1,2, . .),
$$

then the mode is non-pulsed.
The complex form of the scalar IP of the nonsinusoidal mode (36) expands the complex form of the scalar IP of the non-symmetric sinusoidal mode [1]

$$
p(t)=\Re e\left\{\dot{S}_{G_{1}}+\dot{N}_{1} e^{j 2 k \omega t}\right\},
$$

where $\dot{S}_{G_{1}}=\boldsymbol{U}_{1}^{\tau} \boldsymbol{I}_{1}^{*}, \dot{N}_{1}=\boldsymbol{I}_{1}^{\tau} \boldsymbol{U}_{1}-$ complex (geometrical) power and complex pulsation power of current and voltage fundamental harmonics.

Vectorial instantaneous power of the nonsinusoidal mode. Because of the linearity of the vector product, the vector IP

$$
\boldsymbol{q}(t)=\boldsymbol{i} \times \boldsymbol{u}=\sum_{k, m} \underbrace{\boldsymbol{i}_{k} \times \boldsymbol{u}_{m}}_{\boldsymbol{q}_{k, m}(t)}=\sum_{k, m} \boldsymbol{q}_{k, m}(t)
$$

equals to the sum of vector products of the current harmonics of the $k$-th order and voltage harmonics of the $m$-th order

$$
\boldsymbol{q}_{k, m}(t)=\mathfrak{R} e[\underbrace{\boldsymbol{I}_{k} \times \boldsymbol{U}_{m}^{*}}_{\boldsymbol{D}_{k-m}} e^{j(k-m) \omega t}+\underbrace{\boldsymbol{I}_{k} \times \boldsymbol{U}_{m}}_{\boldsymbol{D}_{k+m}} e^{j(k+m) \omega t}]
$$

If the current and voltage harmonics have the same order $m=k$, then their VP has both constant and variable components

$$
\boldsymbol{q}_{k}(t)=\mathfrak{R} e[\underbrace{\boldsymbol{I}_{k} \times \boldsymbol{U}_{k}^{*}}_{\boldsymbol{K}_{k}}+\underbrace{\boldsymbol{I}_{k} \times \boldsymbol{U}_{k}}_{\boldsymbol{D}_{k}} e^{j 2 k \omega t}] .
$$

The complex form of the vector IP is represented by the 3-phasors of the harmonics as

$$
\begin{aligned}
& \boldsymbol{q}(t)=\mathfrak{R} e\left\{\sum_{k \geq 1}\left[\boldsymbol{K}_{k}+\boldsymbol{D}_{k} e^{j 2 k \omega t}\right]\right\}+ \\
& +\mathfrak{R} e\left\{\sum_{k \neq m}\left[\boldsymbol{D}_{k-m} e^{j(k-m) \omega t}+\boldsymbol{D}_{k+m} e^{j(k+m) \omega t}\right]\right\} .
\end{aligned}
$$

The 3-phasors of the current and voltage harmonics' balanced power of the $k$-th order

$$
\boldsymbol{K}_{k}=\boldsymbol{I}_{k} \times \boldsymbol{U}_{k}^{*}(k=1,2, \ldots)
$$

determine the constant component of the vector IP.

The variable (pulsed) component of the vector IP is determined by 3-phasors of the power of the unbalance:

- of the current and voltage harmonics of the $k$-th order of the twice frequency $2 \kappa \omega$

$$
\boldsymbol{D}_{k}=\boldsymbol{I}_{k} \times \boldsymbol{U}_{k}(k=1,2, \ldots) ;
$$

- of the current harmonics of the $k$-th order and the voltage harmonics of the $m$-th order of the sum and difference frequency $(k, m=1,2, \ldots)$

$$
\boldsymbol{D}_{k+m}=\boldsymbol{I}_{k} \times \boldsymbol{U}_{m}, \boldsymbol{D}_{k-m}=\boldsymbol{I}_{k} \times \boldsymbol{U}_{m}^{*} .
$$

If 3-phasors (49) equal to zero

$$
\boldsymbol{D}_{k}=\boldsymbol{D}_{k+m}=\boldsymbol{D}_{k-m}=0,
$$

than the mode is balanced.

If in addition to the conditions (50) the following condition is fulfilled

$$
\mathfrak{R} e \boldsymbol{K}_{k}=\mathfrak{R} e\left[\boldsymbol{I}_{k} \times \boldsymbol{U}_{k}^{*}\right]=0 \quad(k=1,2 \ldots),
$$

then the mode is really balanced.

The complex form of the vector instantaneous power of the non-sinusoidal mode (47) expands the complex form of the vector IP of the sinusoidal non-symmetrical mode [1]

$$
\boldsymbol{q}(t)=\mathfrak{R} e\left\{\boldsymbol{K}_{1}+\boldsymbol{D}_{1} e^{j 2 \omega t}\right\},
$$


where $\boldsymbol{K}_{1}=\boldsymbol{I}_{1} \times \boldsymbol{U}_{1}^{*}, \quad \boldsymbol{D}_{1}=\boldsymbol{I}_{1} \times \boldsymbol{U}_{1}$ are 3-phasors of the balanced power and the power of the unbalance of the current and voltage fundamental harmonic.

Equations of the non-sinusoidal mode's complex powers. For the square of the (apparent) total power the following equality is true

$$
S^{2}=\left(\sum_{k=1}^{\infty} U_{k}^{2}\right) \cdot\left(\sum_{m=1}^{\infty} I_{m}^{2}\right)=\sum_{m=k}^{\infty} I_{m}^{2} \cdot U_{m}^{2}+\sum_{m \neq k}^{\infty} I_{m}^{2} U_{k}^{2} .
$$

The connection between the scalar and vector products of 3-phasors $\boldsymbol{X}, \boldsymbol{Y} \in C^{(3)}$ is determined by the identity [1]

$$
|\boldsymbol{X}|^{2}|\boldsymbol{Y}|^{2}=\left|\boldsymbol{X}^{\tau} \boldsymbol{Y}^{*}\right|^{2}+|\boldsymbol{X} \times \boldsymbol{Y}|^{2},
$$

which expands the corresponding identity of vector algebra of real vectors [2].

At $\boldsymbol{X}=\boldsymbol{U}_{k}, \boldsymbol{Y}=\boldsymbol{I}_{m}$ from the identity (53) the following equalities follow

$$
I_{m}^{2} U_{k}^{2}=\left|\boldsymbol{U}_{k}\right|^{2}\left|\boldsymbol{I}_{m}\right|^{2}=|\underbrace{\boldsymbol{U}_{k}^{\tau} \boldsymbol{I}_{m}^{*}}_{\dot{N}_{k-m}}|^{2}+|\underbrace{\boldsymbol{U}_{k} \times \boldsymbol{I}_{m}}_{\boldsymbol{D}_{k+m}}|^{2},
$$

which at $m=k$ give

$$
I_{k}^{2} U_{k}^{2}=\left|\boldsymbol{U}_{k}\right|^{2}\left|\boldsymbol{I}_{k}\right|^{2}=|\underbrace{\boldsymbol{U}_{k}{ }^{\tau} \boldsymbol{I}_{k}}_{\dot{S} G_{k}}{ }^{*}|^{2}+|\underbrace{\boldsymbol{U}_{k} \times \boldsymbol{I}_{k}}_{\boldsymbol{D}_{k}}|^{2} .
$$

Expansion of the square of the apparent power (52) taking into account (54), (55) gives an equation for the complex scalar and vector powers including active (37) and reactive (40) powers of the sinusoidal mode

$$
S^{2}=\sum_{k=1}^{\infty}\left(P_{k}^{2}+Q_{k}^{2}+D_{k}^{2}\right)+\sum_{m \neq k}^{\infty}\left(N_{k-m}^{2}+D_{k+m}^{2}\right) .
$$

The power equation (56) generalizes the power equation for the sinusoidal non-symmetrical mode [1]

$$
S^{2}=P_{1}^{2}+Q_{1}^{2}+D_{1}^{2} \text {. }
$$

The equation (56) includes not all complex scalar and vector powers of the complex form of the scalar (36) and vector (47) IP.

If to use a couple of sequences $\left\{\boldsymbol{U}_{k}\right\}_{k \geq 1},\left\{\boldsymbol{I}_{m}^{*}\right\}_{m \geq 1}$ then an additional equation for the complex scalar and vector powers which are not included in (56) is true. At $\boldsymbol{X}=\boldsymbol{U}_{k}, \boldsymbol{Y}=\boldsymbol{I}_{m}^{*}$ from the identity (53) the following equalities follow

$$
\begin{gathered}
\left|\boldsymbol{U}_{k}\right|^{2}\left|\boldsymbol{I}_{m}\right|^{2}=|\underbrace{\boldsymbol{U}_{k}{ }^{\tau} \boldsymbol{I}_{m}}_{\dot{N}_{k+m}}|^{2}+|\underbrace{\boldsymbol{U}_{k} \times \boldsymbol{I}_{m}^{*}}_{\boldsymbol{D}_{k-m}}|^{2} . \\
\text { At } m=k \text { equalities }(58) \text { give } \\
\left|\boldsymbol{U}_{m}\right|^{2}\left|\boldsymbol{I}_{m}\right|^{2}=|\underbrace{\boldsymbol{U}_{m}{ }^{\tau} \boldsymbol{I}_{m}}_{\dot{N}_{m}}|^{2}+|\underbrace{\boldsymbol{U}_{m} \times \boldsymbol{I}_{m}^{*}}_{\boldsymbol{K}_{m}}|^{2} .
\end{gathered}
$$

Expansion of the square of the apparent power (52) taking into account (58), (59) gives an additional equation for the complex scalar and vector powers

$$
S^{2}=\sum_{k=1}^{\infty}\left(K_{m}^{2}+N_{m}^{2}\right)+\sum_{m \neq k}^{\infty}\left(N_{k+m}^{2}+D_{k-m}^{2}\right) .
$$

It is possible to show that for each harmonic the following implication is true

$$
\boldsymbol{D}_{m}=0 \Rightarrow K_{m}^{2}+N_{m}^{2}=P_{m}^{2}+Q_{m}^{2}(k=1,2 \ldots) .
$$

The obtained power equation (60) generalizes the additional equation for the sinusoidal non-symmetrical mode [1]

$$
S^{2}=K_{1}^{2}+N_{1}^{2}
$$

As shown in [1] at the sinusoidal mode equations (57), (61) are determined by two different orthogonal expansions of the 3-phase current. The problem of building the current's orthogonal expansion at the sinusoidal mode is used for the solution of the problem of the apparent power's non-active components compensation [3]. Building the orthogonal expansion which is associated with power equations (56), (60) is not considered in this paper and required additional investigations.

The practical value of the obtained equations is a possibility of their utilization for the increase both quality of delivery and quality of consumption of electrical energy.

Conclusion. For the 3-phase 4-wire network with non-sinusoidal (independently on their symmetry) processes the complex forms of the active (scalar) and (nonactive) vector IP are obtained. The power equations for complex scalar and complex vector powers of nonsinusoidal modes are obtained. The power equations generalize the power equations for sinusoidal modes in the 4-wire network.

\section{REFERENCES}

1. Sirotin Iu.A. Vectorial instantaneous power and energy modes in three-phase circuits. Tekhnichna elektrodynamika Technical electrodynamics, 2013, no.6, pp. 57-65. (Rus).

2. Korn G., Korn T. Spravochnik po matematike dlia nauchnykh rabotnikov $i$ inzhenerov [Mathematical handbook for scientists and engineers]. Moscow, Nauka Publ., 1973. 832 p. (Rus).

3. Sirotin Iu.A. Non-pulsed mode of supply in a three-phase system at asymmetrical voltage. Przeglad Elektrotechniczny, 2013, no.7, pp. $54-58$.

Received 16.10.2015

Iu.A. Sirotin ${ }^{1}$, Doctor of Technical Science, Professor,

T.S. Ierusalimova ${ }^{1}$, Assistant Lecturer,

${ }^{1}$ National Technical University «Kharkiv Polytechnic Institute», 21, Frunze Str., Kharkiv, 61002, Ukraine.

e-mail: yuri_sirotin@ukr.net, Ierusalimovat@mail.ru

How to cite this article:

Sirotin Iu.A., Ierusalimova T.S. Instantaneous and integral power equations of nonsinusoidal 3-phase processes. Electrical engineering \& electromechanics, 2016, no.1, pp. 69-73. doi: 10.20998/2074-272X.2016.1.13. 